\title{
Healthcare Payment Method
}

National Cancer Institute

\section{Source}

National Cancer Institute. Healthcare Payment Method. NCI Thesaurus. Code C70680.

Methodology used to compensate a healthcare provider for care provided to a patient. 